split up into a great number of fine pencils. In first approximation, each one of these pencils is collimated in itself; that is to say, within each mesh the lens effect produced by $E$ is exactly compensated. But the global or macroscopic concentrating effect is by no means destroyed, as the central rays of the pencils suffer no deflexion. The effect of replacing the foil by a grid is merely that of replacing the point focus by a cross-over, which to a first approximation has the size and shape of a single grid hole. The next higher approximations (finite depth of the grid, lens effect quadratic in $E$, and spherical aberration) have all signs which point in a beneficial direction, that is to say, they tend to make the cross-over even smaller than a mesh hole.

With modern techniques it is possible to produce slat grids of a great variety of metals with clear areas of 98 and even 99 per cent, which can stand beam energies of several hundred watts per $\mathrm{cm}^{2}$. The danger of auto-electronic discharges starting from the sharp edges of the slats need not be very serious, as sufficient stability of operation can be obtained if the average field intensity $E$ over the surface of the grid is only 10 per cent of the maximum or even less. Thus it appears that grid lenses may well compete with beryllium foils in the stabilization of linear accelerators for extreme energies.

I am indebted to Prof. Oliphant for his suggestion, and to the directors of the British Thomson-Houston Company for permission to publish this note.

D. GABOR

Research Laboratory,

British Thomson-Houston Co., Ltd., Rugby. Jan. 5.

\section{A Simple Film Balance for Demonstration and Research Purposes}

THe Langmuir-Adam film balance was developed to measure the lowering of surface tension (termed the surface pressure) produced by paraffin-chain compounds such as oleic acid when spread as monolayers on aqueous solutions. With substances giving condensed films (for example, stearic acid, cetyl alcohol) the sudden lowering of surface tension when the molecules are squeezed into close-packing provides a striking lecture demonstration, and many simple experiments illustrating molecular orientation, changes of phase and physical state in two-dimensions, etc., can be demonstrated by this means. In addition, the film balance has been widely applied for research purposes, particularly to problems of colloidal systems, such as foams, emulsions, proteins and polymers ; to structure determination of complex organic molecules, and recently to surface-area determination of fine powders by adsorption of longchain polar compounds from organic solvents.

To cater for the greatly increased interest in surface chemistry, the present type of film balance was devised. In addition to simplicity of construction it has some advantages over the usual type employing a torsion balance. The principle can be seen from the accompanying figure.

The monolayer is merely compressed by the usual waxed glass slide against a relatively stout torsion wire, or better, a thin metal strip (the use of a strip is preferred as it automatically brings the float to a fixed position), and the resulting deflexion of the float amplified optically as shown. The reflecting mirror shown at the top of the figure is located by means

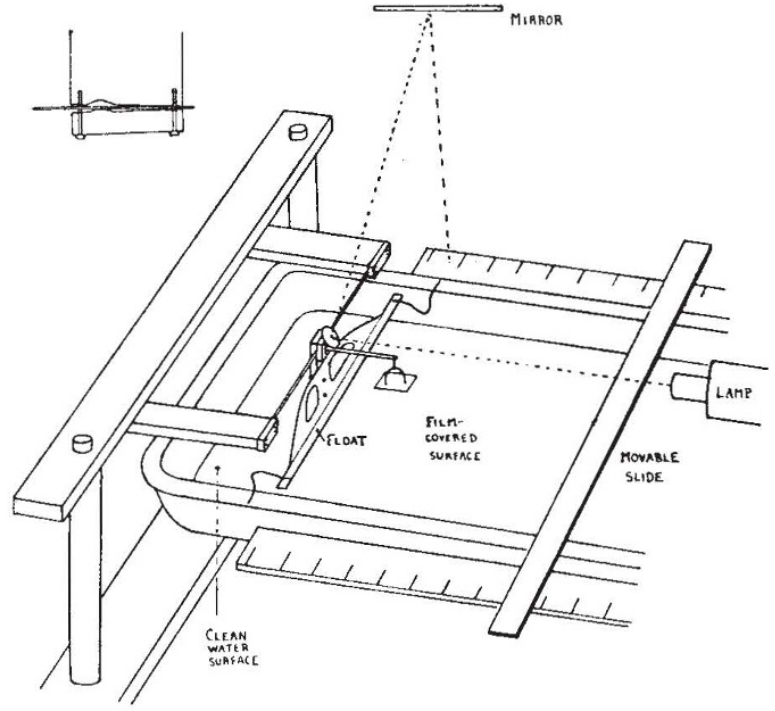

of a tall stand almost vertically above the far end of the torsion arm. A two-metre optical path has been found convenient for ordinary purposes. One of the horizontal scales used to indicate the position of the movable barrier also serves to measure the optical deflexions and hence the force on the float. Errors in film area due to the movement of the float are usually negligible (they can be allowed for if necess. ary), since this movement is deliberately kept very small. With a distance of $2 \mathrm{~cm}$. between the torsion wire (strip) and the float, $1 \mathrm{~mm}$. movement corresponds to $20 \mathrm{~cm}$. deflexion at 2 metres. The torsion wire or strip is suitably chosen so that the float movement does not exceed c.' $1 \mathrm{~mm}$. for the maximum surface pressures encountered. Thin steel watch spring has been found very suitable for the usual film pressures, that is, of the order 10-50 dynes $/ \mathrm{cm}$. The torsion wire or strip can readily be changed merely by loosening the simple clips at its ends, and no difficulty in tightening it sufficiently has so far been encountered, but if necessary the device shown inset can be used.

The float consists of thin duralumin (or other metal) sheet, turned up at the edge and screwed to the vertical duralumin bar to which the torsion wire or strip is clamped. For research purposes a thin mica strip is attached by wax beneath the float to keep all metal out of contact with the solution. A rectangular 'Pyrex' baking-dish, or the glass dish used in 'Frigidaire' refrigerators, provides an excellent trough after the bevelled edges have been ground flat.

Calibration can be carried out in the usual way by adding weights to the small pan (a light wire ring and piece of paper) shown in the figure, or by spreading 'piston oils', such as castor oil or oleic acid, which give known surface pressures.

This apparatus appears to give an accuracy fully equal to that of the usual torsion balance, with the additional advantage that measurements can be carried out much more rapidly. It can also be used for automatic recording of force-area curves, and in addition can readily be adapted to measure surface or interfacial tensions.

Department of Colloid Science,

A. E. Alexander 